\title{
Complex phase dynamics in coupled bursters
}

\section{Postnov, D.E.; Sosnovtseva, Olga; Malova, S.Y.; Mosekilde, Erik}

Published in:

Physical Review E. Statistical, Nonlinear, and Soft Matter Physics

Link to article, DOI:

10.1103/PhysRevE.67.016215

Publication date:

2003

Document Version

Publisher's PDF, also known as Version of record

Link back to DTU Orbit

Citation (APA):

Postnov, D. E., Sosnovtseva, O., Malova, S. Y., \& Mosekilde, E. (2003). Complex phase dynamics in coupled bursters. Physical Review E. Statistical, Nonlinear, and Soft Matter Physics, 67(1), 016215.

https://doi.org/10.1103/PhysRevE.67.016215

\section{General rights}

Copyright and moral rights for the publications made accessible in the public portal are retained by the authors and/or other copyright owners and it is a condition of accessing publications that users recognise and abide by the legal requirements associated with these rights.

- Users may download and print one copy of any publication from the public portal for the purpose of private study or research.

- You may not further distribute the material or use it for any profit-making activity or commercial gain

- You may freely distribute the URL identifying the publication in the public portal

If you believe that this document breaches copyright please contact us providing details, and we will remove access to the work immediately and investigate your claim. 


\title{
Complex phase dynamics in coupled bursters
}

\author{
D. E. Postnov, ${ }^{1}$ O. V. Sosnovtseva, ${ }^{1,2}$ S. Y. Malova, ${ }^{1}$ and E. Mosekilde ${ }^{2}$ \\ ${ }^{1}$ Physics Department, Saratov State University, Astrakhanskaya Street 83, Saratov 410026, Russia \\ ${ }^{2}$ Department of Physics, The Technical University of Denmark, 2800 Kongens Lyngby, Denmark
}

(Received 7 October 2002; published 29 January 2003)

\begin{abstract}
The phenomenon of phase multistability in the synchronization of two coupled oscillatory systems typically arises when the systems individually display complex wave forms associated, for instance, with the presence of subharmonic components. Alternatively, phase multistability can be caused by variations of the phase velocity along the orbit of the individual oscillator. Focusing on the mechanisms underlying the appearance of phase multistability, the paper examines a variety of phase-locked patterns in the bursting behavior of a model of coupled pancreatic cells. In particular, we show how the number of spikes per train and the proximity of a neighboring equilibrium point can influence the formation of coexisting regimes.
\end{abstract}

DOI: 10.1103/PhysRevE.67.016215

PACS number(s): $05.45 . \mathrm{Xt}$

\section{INTRODUCTION}

Bursting is the primary mode of electrical activity for a variety of nerve and endocrine cells [1]. Braun et al. [2], for instance, have investigated bursting patterns in discharging cold fibers of the cat, and Braun et al. [3] have studied the effect of noise on signal transduction in shark sensory cells. Plant and Kim [4] have developed a mathematical model to account for experimentally observed burst patterns in pacemaker neurons, and Morris and Lecar [5] have modeled the complex firing patterns in barnacle giant muscle fibers.

It is known that pancreatic $\beta$ cells under normal circumstances display a bursting behavior with alternations between an active (spiking) state and a silent state [6]. It is also established [7] that the secretion of insulin depends on the fraction of time that the cells spend in the active state, and that this fraction increases with the concentration of glucose in the extracellular environment. The bursting dynamics controls the influx of $\mathrm{Ca}^{2+}$ ions into the cell, and calcium is considered an essential trigger for the release of insulin. In this way, the bursting dynamics serves to organize the response of the $\beta$ cells to varying glucose concentrations. At glucose concentrations below $5 \mathrm{mM}$, the cells remain silent. For very high glucose concentrations $(>22 \mathrm{mM})$, on the other hand, the cells spike continuously, and the secretion of insulin saturates [8]. To provide the total output of a pancreatic islet, a large number of $\beta$ cells must cooperate. When interaction between the individual $\beta$ cells is strong, sizable clusters of cells operate as a single unit. With weaker interaction, however, the mutual entrainment of the firing activities of the coupled cells becomes less trivial.

Many processes in nature may be characterized by a significant number of coexisting regimes at a given set of parameters but with different initial conditions. A set of possible operating regimes serves as an example of multistability. We focus on the phenomenon of phase multistability, i.e., the simultaneous existence of stable synchronous regimes with different phase relationships between the oscillations. Originally, this type of multistability was observed for diffusively coupled functional units that individually follow the Feigenbaum period-doubling route to chaos [9-11]. The possible synchronous regimes increase in number when more subharmonics of the basic frequency can be distinguished in the power spectrum. Phase multistability also takes place for weak chaos that demonstrates an $N$-band structure.

The hierarchy of multistability in identical interacting systems with weak dissipative coupling was studied numerically and experimentally by Astakhov et al. [9]. Vadivasova et al. [10] showed that this type of multistability is structurally stable with respect to a weak mismatch between the basic frequencies. However, the bifurcation sequence changes when the mismatch or the coupling strength is increased. Postnov et al. [11] described the nested structure of the phase synchronized regions. Recently, considering a model of coupled units in the kidney, similar results were obtained for self-modulated oscillations with multicrest wave forms [12]. For a system of two diffusively coupled oscillators operating in the $1: n$ regime of self-modulation ( $n$ being an integer), one expects $n$ coexisting synchronous solutions that differ from one another by phase shifts. The corresponding synchronization region consists of a set of $n$ Arnol'd tongues embedded one into the other or shifted with respect to the mean synchronization frequency.

Diffusive coupling between two limit cycle oscillators typically leads to in-phase synchronization being the only stable state in the weak-coupling limit $[13,14]$. It was recently shown, however, that the same coupling for MorrisLecar neuron models [15] and modified Van der Pol oscillators [16] can give rise to antiphase synchronization when the limit cycle is close to a homoclinic bifurcation. In this case, the dephasing originates from a deformation of the phase flow, i.e., from a strong variation of the phase velocity along the limit cycle.

To our knowledge, the multistability of bursting oscillators has not yet been examined in detail. de Vries et al. [17] found asymmetrically phase-locked solutions to be typical for coupled heterogeneous beta cells while a set of coexisting out-of-phase regimes was observed for coupled HindmarshRose models $[18,19]$. When changing the initial conditions the latter system switched from one burst-locked mode to another with fixed parameters. In the present paper, instead of delineating different regimes and following the evolution of various coexisting solutions, we focus on different mechanisms of phase complexity in coupled bursters described by the Sherman model [20]. These mechanisms are related to 

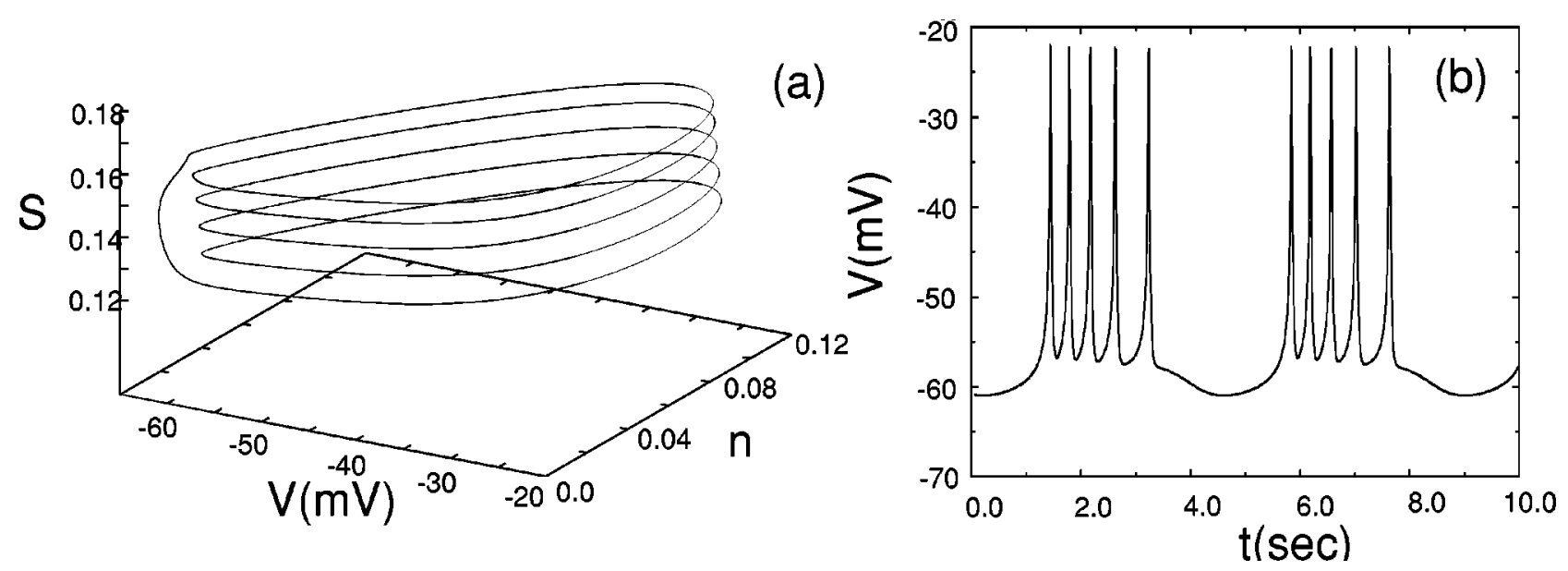

FIG. 1. Example of bursting oscillations in the single Sherman model with five spikes per burst. (a) 3D phase plot; (b) temporal variation for the membrane potential $\left(V_{S}=-39.0 \mathrm{mV}, k_{S}=0.00057\right)$.

the complex wave forms of the oscillations as well as to a variant of the above mentioned dephasing effect. As a quantitative measure of phase dynamics we use the method of effective coupling [21] that provides information on the phase properties of the interacting solutions and on the number of synchronous regimes.

\section{MODEL}

As a basis for the present analysis we use the simplified model of a pancreatic $\beta$ cell suggested by Sherman et al. [22]:

$$
\begin{gathered}
\tau \frac{d V}{d t}=-I_{\mathrm{Ca}}(V)-I_{\mathrm{K}}(V, n)-I_{S}(V, S), \\
\tau \frac{d n}{d t}=\lambda\left[n_{\infty}(V)-n\right], \\
\tau_{S} \frac{d S}{d t}=S_{\infty}(V)-S,
\end{gathered}
$$

where

$$
\begin{gathered}
I_{\mathrm{Ca}}(V)=g_{\mathrm{Ca}} m_{\infty}(V)\left(V-V_{\mathrm{Ca}}\right), \\
I_{\mathrm{K}}(V)=g_{\mathrm{K}} n\left(V-V_{\mathrm{K}}\right), \\
I_{S}(V)=g_{S} S\left(V-V_{\mathrm{K}}\right),
\end{gathered}
$$$$
\omega_{\infty}(V)=\frac{1}{1+\exp \left[\left(V_{\omega}-V\right) / \Theta_{\omega}\right]} \text { with } \omega=m, n \text {, and } S \text {. }
$$

Here, $V$ represents the membrane potential while $n$ may be interpreted as the opening probability of the potassium channels and $S$ accounts for the presence of a slow dynamics in the system. $S$ is likely to be related to the intracellular $\mathrm{Ca}^{2+}$ concentration, although the precise biophysical interpretation of this variable remains unclear. $I_{\mathrm{Ca}}$ and $I_{\mathrm{K}}$ are the calcium and potassium currents, $g_{\mathrm{Ca}}=3.6$ and $g_{\mathrm{K}}=10.0$ the associated conductances, and $V_{\mathrm{Ca}}=25 \mathrm{mV}$ and $V_{\mathrm{K}}=$
$-75 \mathrm{mV}$ the respective Nernst (or reversal) potentials. $\tau / \tau_{S}$ defines the ratio of the fast ( $V$ and $n$ ) and the slow $(S)$ time scales. The time constant for the membrane potential is determined by the capacitance and the typical total conductance of the cell membrane. With $\tau=0.02 \mathrm{~s}$ and $\tau_{S}=35 \mathrm{~s}$, the ratio $k_{S} \equiv \tau / \tau_{S}$ is quite small, and the cell model is numerically stiff.

The calcium current $I_{\mathrm{Ca}}$ is assumed to adjust instantaneously to variations in $V$. For fixed values of the membrane potential, the gating variables $n$ and $S$ relax exponentially towards their voltage dependent steady state values $n_{\infty}(V)$ and $S_{\infty}(V)$. Together with the ratio $k_{S}$ of the fast to the slow time constant, $V_{S}$ will be used as the main bifurcation parameter. This parameter determines the membrane potential at which the steady state value for the gating variable $S$ attains half its maximum value. The other parameters are $g_{S}$ $=4.0, \quad V_{m}=-20 \mathrm{mV}, \quad V_{n}=-16 \mathrm{mV}, \quad \theta_{m}=12 \mathrm{mV}, \quad \theta_{n}$ $=5.6 \mathrm{mV}, \theta_{S}=10 \mathrm{mV}$, and $\sigma=0.85$. These values are all adjusted so that the model can reproduce experimentally observed time series. In accordance with the formulation used by Sherman et al. [22], all the conductances have been scaled relative to some typical conductance. Hence, we may also consider Eqs. (1) as the model of a cluster of closely coupled $\beta$ cells that share the capacity and conductance of the total membrane area.

Figure 1 provides an example of the variations of $V, n$, and $S$ as obtained by simulating the cell model under conditions where it exhibits bursting behavior. A bifurcation analysis of the single Sherman model shows a variety of different spiking regimes [23]. An example of a two-dimensional bifurcation diagram is presented in Fig. 2. Near the bottom of this figure we observe a Hopf bifurcation curve. Below this curve, the model has one or more stable equilibrium points. Above the curve we find a region of complex behavior delineated by the period-doubling curve $\mathrm{PD}^{1-2}$. Along this curve, the first period-doubling of the continuous spiking behavior takes place. In the heart of the region surrounded by $\mathrm{PD}^{1-2}$ we find an interesting squid-formed structure with arms of chaotic behavior (indicated in black) stretching down towards the Hopf bifurcation curve. Each of the arms of the squid-formed structure separates a region of periodic 


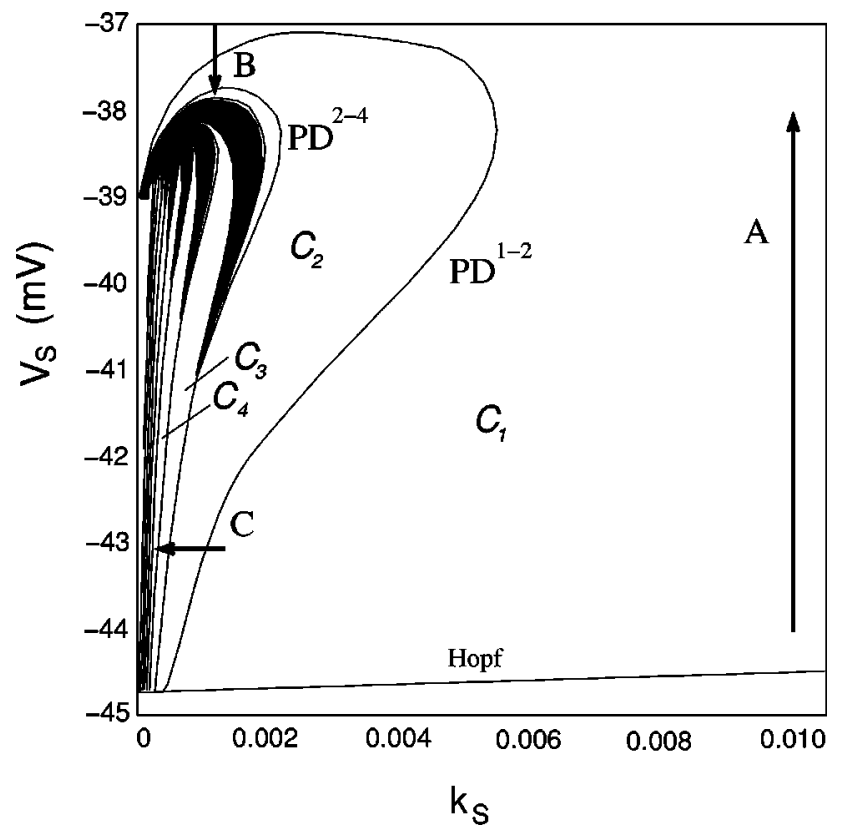

FIG. 2. Two-dimensional bifurcation diagram outlining the main bifurcation structure in the $\left(V_{S}, k_{S}\right)$ parameter plane for the single cell Sherman model. Note the squid-formed black region with chaotic dynamics. Arrows $A, B$, and $C$ indicate different routes of parameter variation discussed in the text.

bursting behavior with $n$ spikes per burst from a region with regular $n+1$ spikes per burst behavior. Each arm has a period-doubling cascade leading to chaos on one side and a saddle-node bifurcation on the other. It is easy to see that the number of spikes per burst becomes large as $k_{S}$ approaches zero.

\section{METHOD}

Since the definition of phase multistability involves the phase difference between the interacting oscillators, the phase variables are the main quantities used to characterize the collective dynamics.

Let us first consider the weak-coupling case, i.e., we assume that the coupling causes only small perturbations of the limit cycles of the uncoupled oscillators. The coupled system may then be approximated by a phase model [21] where the phase $\phi$ of a limit cycle oscillator is defined by $d \phi\left(V_{0}\right) / d t$ $=1$ with $V_{0} \in R^{N}$ being a point on the limit cycle. It is implied that the full length of closed orbit corresponds to $2 \pi$. Applying the concept of isochrons defined stroboscopically (in terms of the period of the stationary oscillation) as a subset of initial conditions that asymptotically converge to the same point on the limit cycle [21], the phase description can be extended to some vicinity of this cycle. Moreover, for a sufficiently small vicinity of the stationary solution one can assume that the above subset is a flat surface that is transversal to the limit cycle at a given point.

In the presence of a small perturbation $P(V)$, the phase dynamics obeys the following equation [21]:

$$
\frac{d \phi}{d t}=1+Z(\phi) P(V)
$$

where the sensitivity function $Z(\phi)=\left.\operatorname{grad}_{V} \phi\right|_{V=V_{0}}$ measures the change of phase along the limit cycle caused by the change of $V$. To derive Eq. (2), we choose a point $V_{0}$ on the limit cycle and a point $V$ close to $V_{0}$ but not on the limit cycle and then measure the difference in phases between $V_{0}$ and $V$. In the limit $\left|V-V_{0}\right| \rightarrow 0$, this difference, divided by $\left|V-V_{0}\right|$, gives the sensitivity function $Z(\phi)$.

The interaction of two identical oscillators with phases $\phi_{1}$ and $\phi_{2}$ can be quantified by the evolution of their phase difference $\Delta \phi=\phi_{1}-\phi_{2}$. In the limit of weak interaction, averaged over a period, the phase dynamics for one of the oscillators can be expressed as [21]

$$
\frac{d(\Delta \phi)}{d t}=\Gamma(\Delta \phi)=\frac{1}{2 \pi} \int_{0}^{2 \pi} d \phi Z(\phi) P(\phi, \Delta \phi),
$$

where $P(\phi, \Delta \phi)=P\left(V_{0}(\phi), V_{0}(\phi+\Delta \phi)\right)$ describes the rate of change of the state vector $V$ of one oscillator due to interaction at the other with a phase difference $\Delta \phi$. The product $Z(\phi) P(\phi, \Delta \phi)$ is the phase shift along the limit cycle for the considered perturbation. Note, that the limit cycles in the two systems are assumed to have similar shapes, i.e., to be topologically conjugated.

For mutually coupled oscillators, the entrainment manifests itself as a mutual phase shift. This can be analyzed purely in terms of the antisymmetric part $\Gamma_{a}(\Delta \phi)$ of the effective coupling function (3) [21]. The zeroes of $\Gamma_{a}(\Delta \phi)$ correspond to the phase-locked synchronous states $(\Delta \phi$ $=$ const $)$ and their stabilities are determined by the slope of $\Gamma_{a}(\Delta \phi)$ at the respective states, i.e., a negative slope implies a stable state, and vice versa. This method of effective coupling has been applied in a number of cases $[15,16,19]$. When the coupling becomes strong enough to modify the geometry of the limit cycle, the phase reduction method can no longer be used. Direct numerical methods should then be applied.

To calculate the effective coupling function, it is necessary to define (i) the equations for the model to be coupled and (ii) the coupling function. We assume that the coupling is of diffusive type and expressed by difference terms of the form $C\left(X_{1}-X_{2}\right)$ where $X_{1}=\left(V_{1}, n_{1}, S_{1}\right)^{T}$ and $X_{2}$ $=\left(V_{2}, n_{2}, S_{2}\right)^{T}$ are the state vectors of the individual cell models. $C$ is the coupling matrix for which we assume the form $C=\operatorname{diag}(1,0,1)$, indicating that coupling takes place via the first and the third variables. The membrane potentials are coupled resistively via electric currents that flow between the cells, and the third variables are coupled via the diffusive exchange of calcium between the cells [23]. The corresponding antisymmetric parts of the effective coupling function are denoted as $\Gamma_{a V}$ and $\Gamma_{a s}$, respectively. We do not consider coupling via the gating variables $n$, since such a coupling appears less realistic from a biological point of view. Note that a coupling strength parameter is absent in the expression for $C$ because the analysis assumes the coupling to be vanishingly weak.

An example of the effective coupling method applied to the Sherman model (1) is illustrated in Fig. 3. Control parameters are the same as in Fig. 1. This implies that each of 

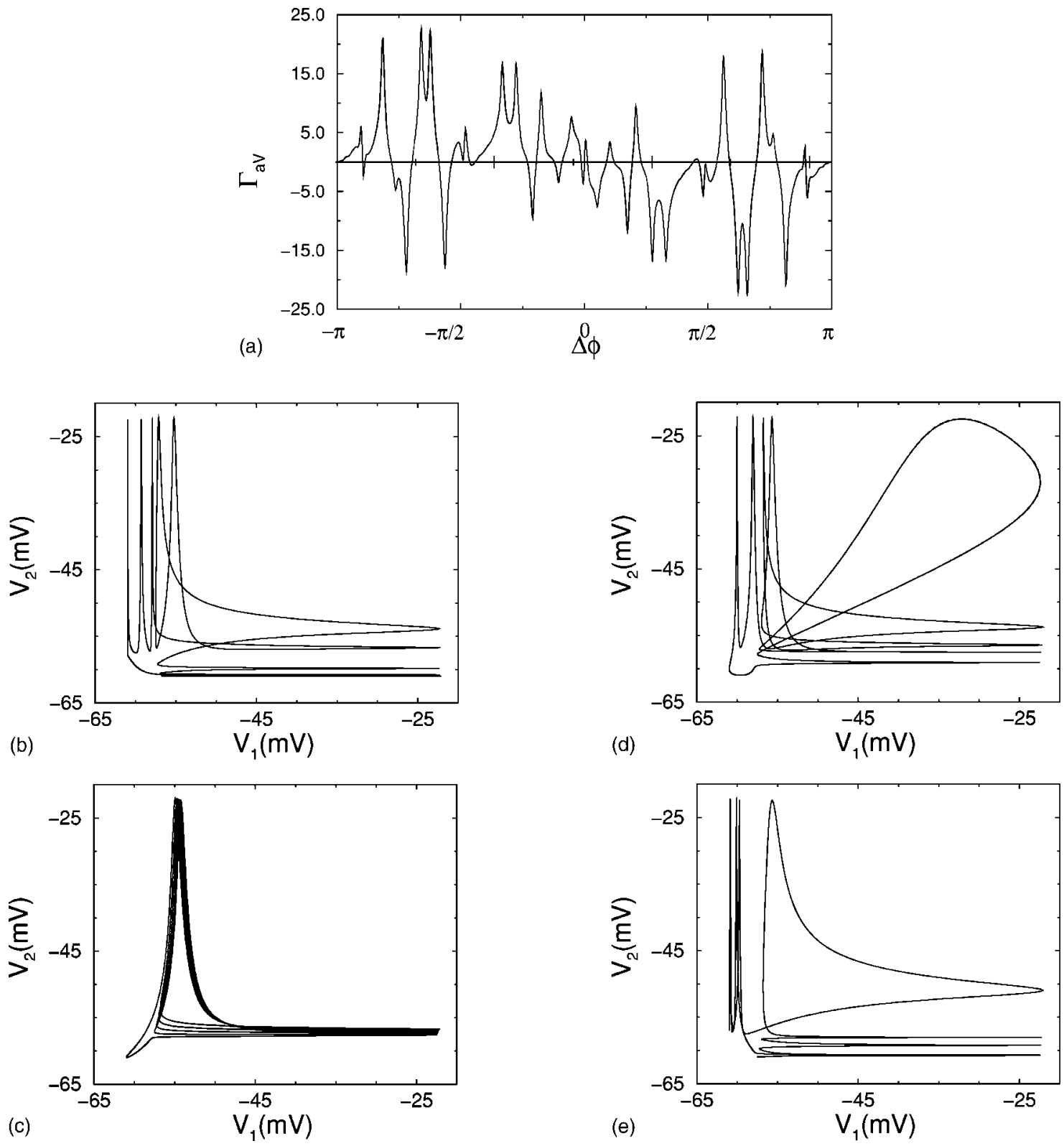

FIG. 3. Upper panel: antisymmetric part $\Gamma_{a V}$ of the effective coupling function calculated for two $V$-coupled Sherman models. A set of 14 stable and 14 unstable synchronous regimes correspond to zero points of the $\Gamma_{a V}$ function. Lower panel contains phase projections for some of the indicated regimes (the coupling strength is 0.0001 ).

the two cell models without coupling perform bursting dynamics with five spikes per burst. $\Gamma_{a}$ reveals a complicated phase behavior for the coupled cell models with a set of coexisting synchronous regimes. Some of the detected regimes, calculated for a finite coupling strength, are depicted in the lower panel. There is no obvious relation between the number of spikes in a train (five) and the detected zero points with negative slope of $\Gamma_{a}$ (fourteen). We conclude that it is difficult to know a priori how many phase-locked regimes one can observe in systems of coupled bursters. To understand the origin of this complexity, the first stage of our investigation will be to describe the phase dynamics of the interacting cells in a state of continuous spiking, i.e., when bursting dynamics has not yet developed.

\section{MULTISTABILITY INDUCED BY DEPHASING}

Let us return to the diagram in Fig. 2. There is no bursting to the right of the curve $\mathrm{PD}^{1-2}$. Here, continuous spiking is the only stable mode. This regime is similar in many ways to the behavior of 2D models, such as the van der Pol oscillator. Thus, a relatively simple pattern for the mutual synchronization of the cells is expected. For van der Pol oscillators, it has been shown that only the in-phase synchronous regime is stable for weak diffusive coupling $[13,14]$. However, inspection of the considered parameter region for coupled Sherman models shows that different patterns of synchronous states can be found for weak diffusive coupling. For example, both in-phase and antiphase regimes can be stable, and an additional pair of out-of-phase solutions can occur. 
As mentioned in the Introduction, there is an interesting mechanism that can produce out-of-phase behavior in weakly coupled oscillatory units. Dephasing has been shown to be responsible for antiphase synchronization in coupled Morris-Lecar neuron models and in coupled modified van der Pol systems $[15,16]$. Although specific in details, models exhibiting this effect have a common structure of their phase space. The presence of a saddle equilibrium located near but outside the limit cycle is crucial. In this case there is an inverse gradient of phase velocity across to the trajectory on the limit cycle. When perturbed by coupling, the phase trajectories of the interacting units can be shifted towards or away from the saddle point and, hence, the dynamics can be slowed or become faster.

In contrast to the above 2D oscillators, the Sherman model has a single equilibrium point inside the limit cycle. How can dephasing arise in this case? We propose that the mutual location of the equilibrium point and a limit cycle in the original Sherman model is responsible for the observed effects. In some region of phase space, the phase trajectory in the single cell model approaches the unstable equilibrium point quite closely. Thus, a weak perturbation can slow down or accelerate the motion of the phase point considerably. This causes the dephasing effect to arise.

To check the above hypothesis, we reduce the model equations (1) to a 2D model with only one fast $(V)$ and one slow $(S)$ variable (i.e., we assume the relaxation of the gating variable $n$ to be very fast.) This produces a model similar to the FitzHugh-Nagumo model in the general form:

$$
\begin{gathered}
\tau \frac{d V}{d t}=-I_{\mathrm{Ca}}(V)-I_{\mathrm{K}}\left(V, n_{\infty}\right)-I_{S}(V, S)=f(V, S), \\
\tau_{S} \frac{d S}{d t}=S_{\infty}(v)-S=g(V, S) .
\end{gathered}
$$

Here the terms are the same as in Eq. (1), but $n_{\infty}$ is used instead of $n$ in the expression for $I_{\mathrm{K}}$.

In Fig. 4 the mutual location of the limit cycle (white curve) and the unstable equilibrium point (EP) is illustrated together with contour plots of the phase velocity (solid lines with gray shading). There is an area of slow motion, determined by the location of the cubic shape nullcline $f(V, S)$ $=0$. At the intersection of $f(V, S)=0$ with the other nullcline $g(V, S)=0$ there is a single point (EP) of zero phase velocity. It is clearly seen how the position of EP changes with varying control parameter (route $A$ in Fig. 2), and the sensitivity to a weak perturbation of the limit cycle changes as well. In the right panel of Fig. 4, a deviation from the unperturbed cycle (white curve) should not produce a significant effect, while motion along the limit cycle in the left panel becomes inhomogeneous.

These qualitative observations are confirmed by calculation of the effective coupling function (Fig. 5). At $V_{S}=$ $-38.19 \mathrm{mV}, k_{S}=0.0175$, the equilibrium point is located far from the limit cycle. In this case, the in-phase synchronous regime is stable, but the antiphase solution is unstable [Fig. 5(b)] for weak diffusive coupling via the $V$ and $S$ variables. This behavior is similar to synchronization of coupled van
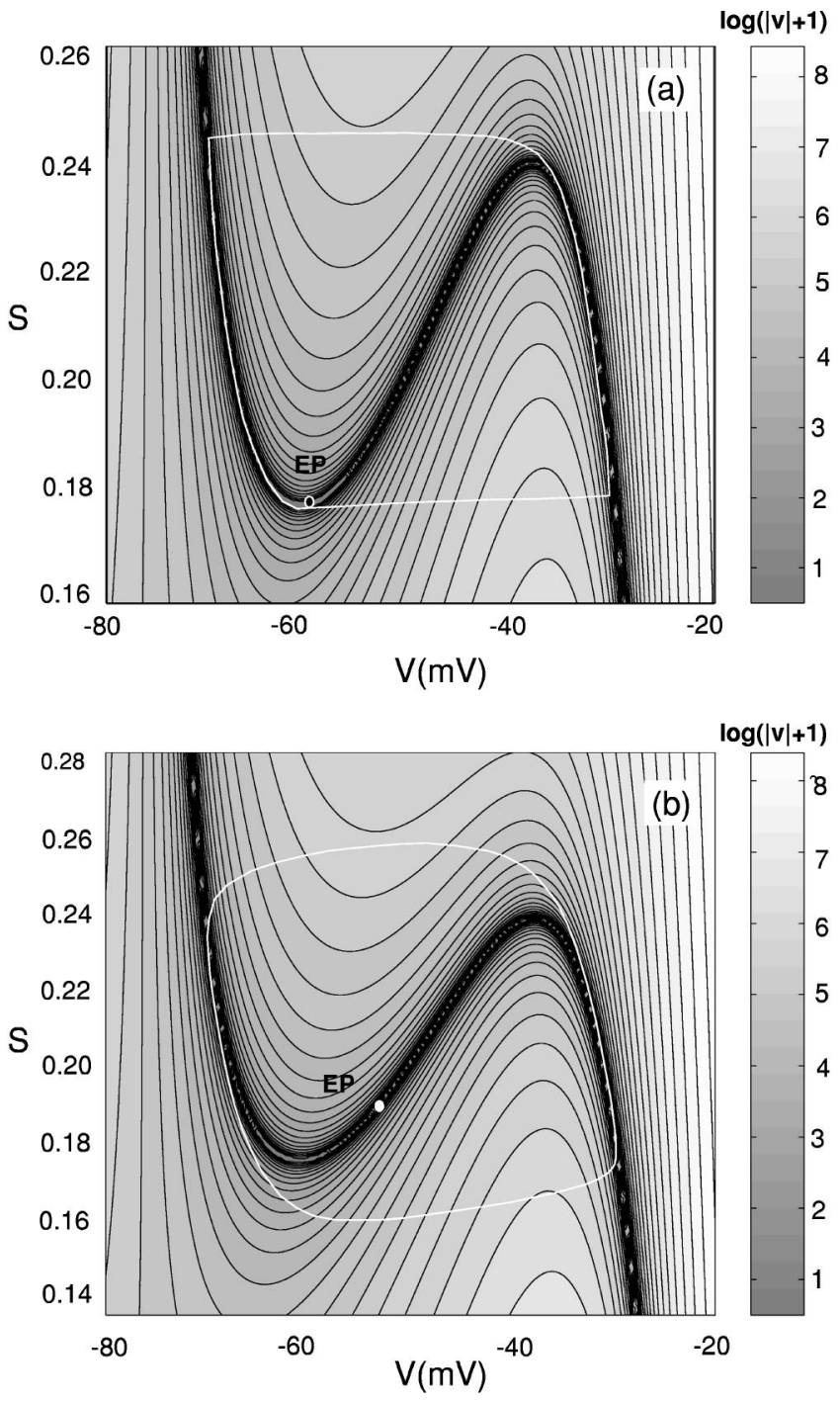

FIG. 4. Phase velocity contour plot for the reduced Sherman model at (a) $V_{S}=-44.0 \mathrm{mV}, k_{S}=0.001$; (b) $V_{S}=-38.19 \mathrm{mV}$, $k_{S}=0.0175$.

der Pol oscillators, and the dephasing effect is not pronounced. As soon as the equilibrium point approaches the limit cycle [Fig. 5(a)], the antiphase regime becomes stable but the in-phase solution maintains its stability in contrast to the dephasing effect described by Han et al. [15]. Two new out-of-phase unstable regimes appear. Simultaneous coupling via both the $V$ and $S$ variables produces a qualitatively similar effect.

Thus, the coupled reduced models (4) exhibit the dephasing effect in a form different from the form described in Refs. $[15,16]$. We expect that the dephasing effect will be preserved when we return to the full Sherman model (1). However, in coupled 3D systems it is difficult to make precise statements about the mutual configuration of a limit cycle and an equilibrium point based on a Poincaré section only. Useful information can be obtained by calculating the distance between the two objects in phase space. In Fig. 6 the variation of the minimal distance between the limit cycle and the equilibrium point is plotted. It is clearly seen that this distance decreases with decreasing values of $V_{S}$. The insets 

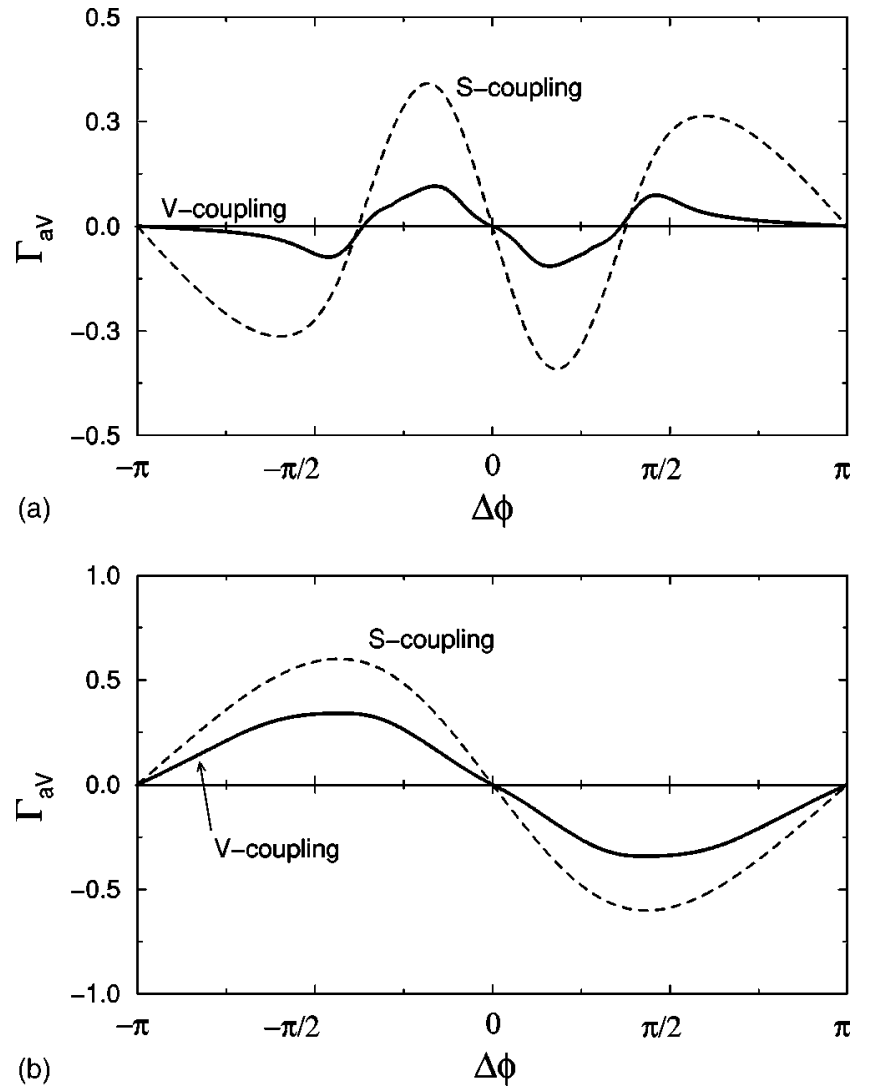

FIG. 5. Antisymmetric part for the effective coupling function, calculated for the reduced Sherman model at (a) $V_{S}=-44.0 \mathrm{mV}$, $k_{S}=0.001$; (b) $V_{S}=-38.19 \mathrm{mV}, k_{S}=0.0175$.

show examples of the $\Gamma_{a}$ shape for selected values of $V_{S}$. For $V_{S}=-38.39 \mathrm{mV}$ the effective coupling function indicates "good" behavior, similar to the behavior observed for coupled van der Pol oscillators. The in-phase state is the only stable solution for coupling via the $V$ (solid line) or $S$ (dashed line) variables. For $V_{S}=-43.25 \mathrm{mV}, \Gamma_{a}$ indicates both inphase and antiphase regimes that are stable both for $S$ coupling and for $V$ coupling.

Summarizing the results of this section, the phase space structure of the Sherman model provides phase multistability even outside of the bursting region. The mechanism for this can be identified as a specific form of dephasing effect, related with a slowing down or acceleration of the trajectory in each coupled unit. Note that the described effect takes place for arbitrary weak coupling and is the result of the phase space properties of the Sherman model rather than of specific features of the coupling. In the bursting area we expect the considered mechanism to interact with the effect of multicrest wave forms, producing additional complexity in the phase patterns.

\section{PHASE MULTISTABILITY DUE TO PERIOD- DOUBLING CASCADE}

The complex wave form appearing due to perioddoubling bifurcations can be considered in terms of fast and slow (lowest over all subharmonics) oscillations being in a

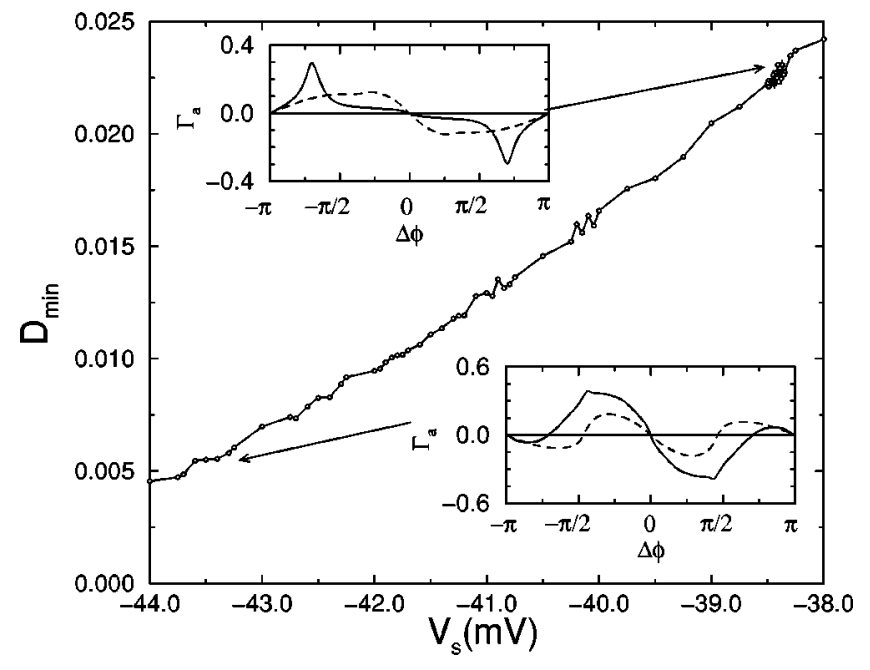

FIG. 6. Minimal distance from the equilibrium point EP to the limit cycle plotted against the value of $V_{S}$ (along the $A$ route in Fig. 2 ). Insets display the qualitatively different responses of the $3 \mathrm{D}$ Sherman model to weak coupling via the $V$ variable (solid line) or the $S$ variable (dashed line). Note, the solid line in the upper inset is reduced by 20 in the vertical scale to fit the same plot as the dashed line.

resonant ratio $1: N$. When the oscillation wave form has $N$ local maxima uniformly distributed over the whole period $T$, there are $N$ values for the phase shift that produce in-phase behavior for the fast oscillations, but a phase-shifted state for all other spectral components. Generally, a stable synchronous regime exists for $N$ values of the phase shift. When the diffusive coupling becomes stronger, symmetry breaking or period-doubling bifurcations can occur and change the number of stable regimes.

For systems demonstrating the period-doubling route to chaos, it was found that the number of synchronous regimes increases when the period of the cycle is increased [9-11]. Let, for simple period-one oscillations with period $T_{0}$, a phase difference between the subsystems be $\phi_{0}$. For oscillations with doubled period whose spectrum contains the subharmonic at frequency $\omega_{0} / 2=1 / 2 T_{0}$, two different limit cycles exist in the phase space of the interacting systems corresponding to the phase differences $\phi_{0} / 2$ and $\left(\phi_{0}\right.$ $+2 \pi) / 2$, respectively. Note that a $2 \pi$ interval of the individual phase now corresponds to the entire period of the limit cycle $2 T_{0}$. For two synchronized oscillators whose spectra include subharmonics $\omega_{0} / 2^{l}(l=1,2, \ldots)$ of the fundamental frequency, the phase difference between the interacting units can attain $2^{l}$ different values distributed over the interval $\quad 0, \ldots, 2 \pi, \quad$ i.e., $\quad \delta \phi=\left(\phi_{0}+2 \pi i\right) / 2^{l}, \quad i$ $=0,1,2, \ldots, 2^{l}-1$. This implies that for a period-two solution one can observe two stable phase-locked regimes while a period-four solution gives rise to four stable synchronous regimes, etc.

To consider the phase dynamics of coupled identical Sherman models (1), we select route $B$ on the parameter plane (Fig. 2). In this area a normal period-doubling cascade can be observed in the synchronization states as the dynamics of the individual model proceeds from continuous spiking towards 
bursting [24]. Figure 7(a) shows the transitions from the period-1 solution (top) to the period-2 solution (bottom) and Fig. 7(b) shows the further development to the period-4 solution. The changes in the location of the zeroes for the $\Gamma_{a V}$ with variation of $V_{S}$ are traced with solid lines. In the period-1 regime, the system demonstrates two stable (filled circles) and two unstable (open circles) coexisting solutions because of the dephasing effect. At $V_{S} \approx-37.35 \mathrm{mV}$, the first period-doubling bifurcation occurs, and the number of coexisting regimes increases. Together with in-phase and antiphase regimes, two stable out-of-phase attractors appear. Note that close to the bifurcation point the values of the phase shift for the stable and unstable regimes are uniformly distributed over the whole interval of $2 \pi$.

A similar transition takes place at the second perioddoubling bifurcation [Fig. 7(b)]. Here, the location of stable and unstable regimes is less regular with respect to the phase difference $\Delta \phi$, but the total number of regimes is doubled at the point of the period-doubling bifurcation.

Thus, the mechanism of phase multistability developed for the period-doubling cascade is applicable to coupled Sherman models as well. However, two specific features can be observed in this case.

(i) Due to the dephasing effect, the original number of regimes differs from the cases studied in previous works. In our case, the first period doubling increases the number of regimes from two to four, instead of from one to two. The second period-doubling bifurcation produces a transition from four to eight regimes, instead of from two to four.

(ii) Immediately after the period-doubling bifurcation, the set of coexisting regimes can be reduced (increased) by other mechanisms (such as dephasing). This is clearly seen near the bottom of Figs. 7(a) and 7(b).

\section{PHASE MULTISTABILITY IN THE BURSTING REGIME}

Bursting dynamics, representing another example of fastand-slow motion, differs from the above described oscillations in the period-doubled regimes since a silent state exists. This implies that local maxima are distributed nonuniformly over the whole period and the set of possible synchronous states is expected to have specific features. Let us develop a simplified qualitative analysis to understand how coexisting regimes arise. The basic assumption for such an analysis is a tendency of coupled units to be synchronized with coincidence of local maxima. The more local maxima (spikes) that coincide, the stronger is presumably the stability of the corresponding regime.

\section{A. Simple qualitative approach}

One can easily count the number of possible regimes with overlapping spikes in two time series (Fig. 8). The results of a more formal analysis can be summarized as follows.

\section{Equidistant spike train}

We consider a signal that is characterized by spiking interval $T_{s p}=n \Delta t$ and silence interval $T_{s}=m \Delta t(n, m$ are integers) with $\Delta t=$ const The whole period is defined as $T$ $=(n+m) \Delta t$.
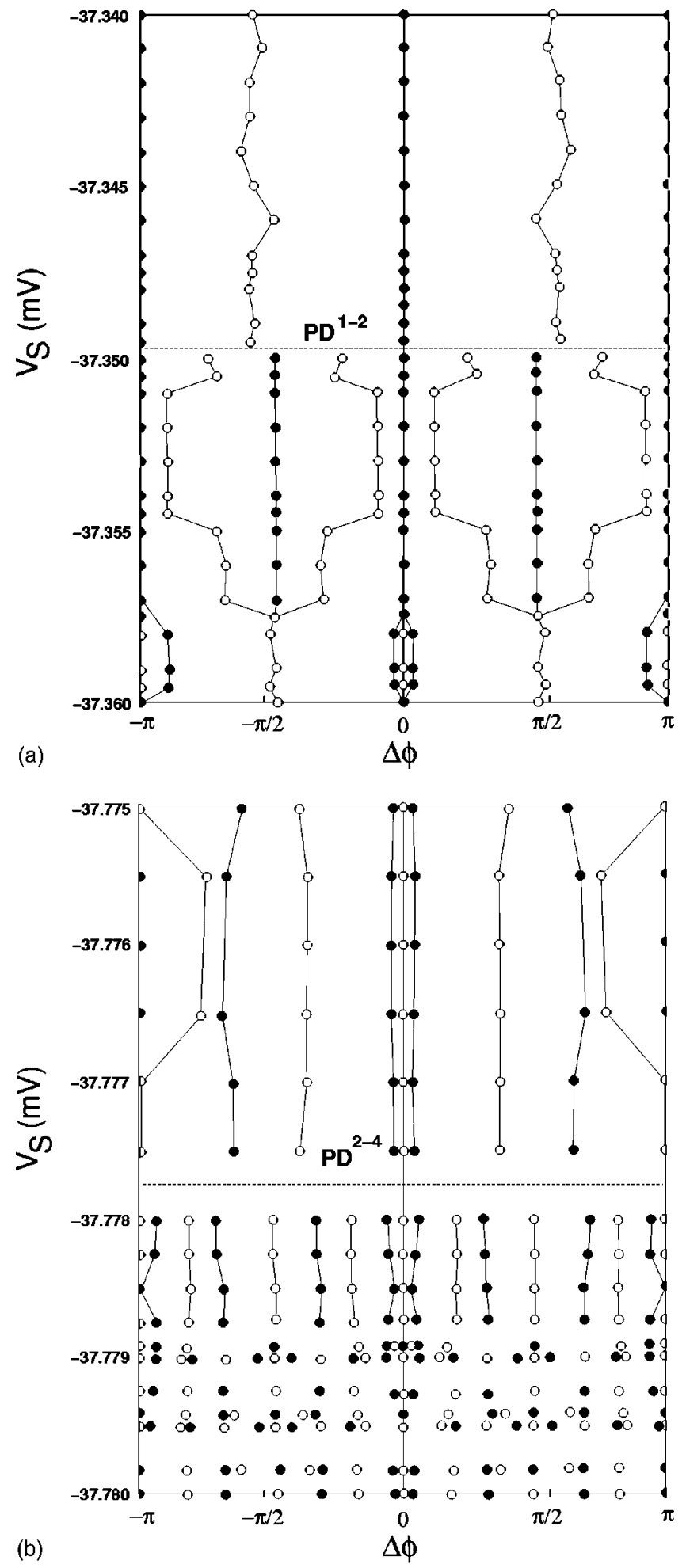

FIG. 7. Schemes of zero point locations for $\Gamma_{a V}$ as $V_{S}$ is varied across the first period-doubling bifurcation $\mathrm{PD}^{1-2}$ (a) and across the second period-doubling bifurcation (b). Black circles denote stable solutions, open circles correspond to unstable regimes.

For two interacting signals $x_{1}(t)$ and $x_{2}(t)$, it is assumed that $n_{1}+m_{1}=n_{2}+m_{2}=N$. To be specific, let $n_{2}<n_{1}$ and, thus, $m_{2}>m_{1}$. 


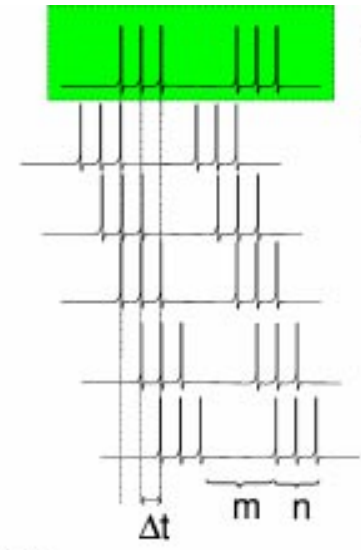

(a)

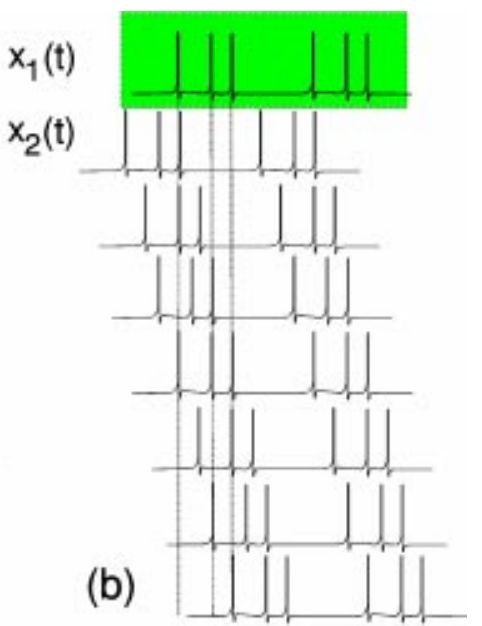

FIG. 8. Sketch of the expected variants of the synchronous regimes for interacting bursting oscillations with three-spike trains. Note the difference between the cases when the interspike distances are equal (a) and different (b).

If $m_{1}<n_{2}-1$, the silent region overlaps with the spike train. Hence, the number of possible combinations is equal to $N_{s}=n_{1}+m_{1}=N$. This coincides with the result for the selfmodulation case [12] or for the period-doubling cascade (for which $m=0$ and the spike amplitudes are different within a burst).

If $m_{1} \geqslant n_{2}-1$, the number of possible synchronous regimes is equal to $N_{s}=n_{1}+n_{2}-1$ and increases with increasing $n_{1}$ and $n_{2}$.

If $n_{1}+m_{1} \neq n_{2}+m_{2}$, but $\Delta t$ still the same for both spike trains, then a minimal period $T_{n m}=\Delta t\left(n_{1}+m_{1}\right)\left(n_{2}+m_{2}\right)$ exists and the problem translates into the previous case. However, the particular configuration of silent regions and spike trains depends on the values of $n_{1}, m_{1}, n_{2}, m_{2}$. The set of synchronous regimes can be estimated as $n_{1}+n_{2}-1$ $\leqslant N_{s} \leqslant\left(n_{1}+m_{1}\right)\left(n_{2}+m_{2}\right)$.

Hence, interacting "perfect" bursting oscillators are expected to provide even more synchronous states than selfmodulated oscillations of the same period $T$.

\section{Nonequidistant spike train}

This case is perhaps more realistic because the typical bursting scenario involves a gradual reduction of the spiking frequency during a burst. In such a situation one can expect the number of coexisting regimes for the interacting bursters to change.

Let one of spikes in the train be located with a different time interval from the other spikes [Fig. 8(b)]. This does not affect the fully in-phase regime. However, the stability of the phase-shifted regimes is likely to become weaker since the coincidence of spikes is not so good as in Fig. 8(a). At the same time, the additional cases of coincidence for the "separated" peak appear. However, even through the tendency to synchronization may not be strong enough to provide additional stable synchronous states, at least they can produce so-called "ghosts" where phase differences develop slowly.

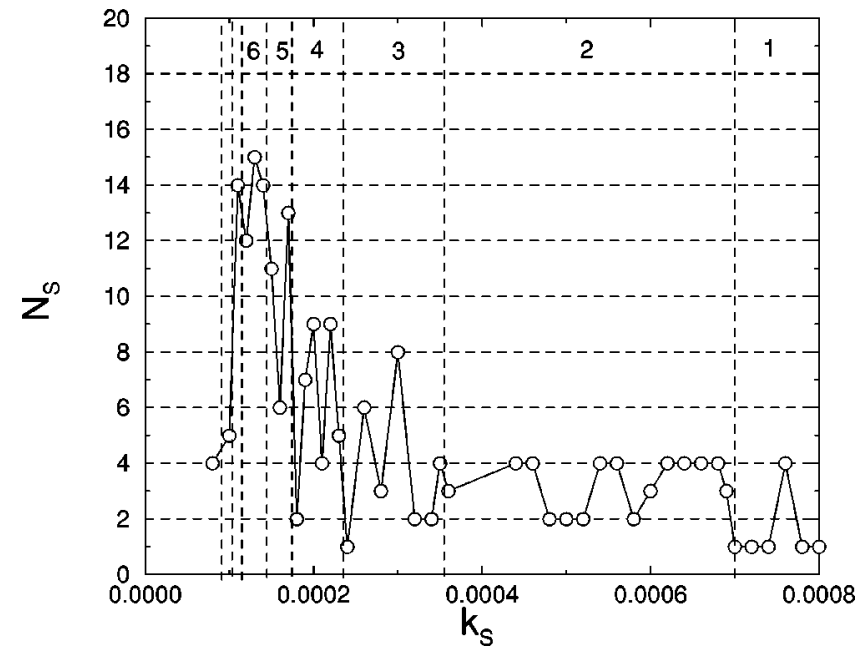

FIG. 9. Number of phase-locked regimes vs the control parameter $k_{S}$ under the period adding scenario $C$ (see Fig. 2). Numbers along the upper edge of the figure denote the number of spikes per burst.

\section{Limitations of the above approach}

In the previous sections we showed that in-phase synchronization is not the only possible state for two coupled Sherman models. Additional synchronous states, anti phase or out of phase, are expected to be stable. It is not clear how the above approach can be extended to the antiphase regime and out-of-phase states. At last, for some regimes the time intervals can be different between all spikes in a train. We conclude that one cannot be sure that an increasing number of coexisting regimes will occur when increasing the spike number per train.

\section{B. Simulation results}

Figure 9 illustrates how the number of detected stable synchronous regimes changes when varying the control parameter $k_{S}$ along the route $C$ as indicated in Fig. 2. Along this route, the number of spikes in a train increases stepwise when crossing the bifurcation curves. The bifurcation mechanism in this direction was described by Mosekilde et al. [20]. One typically observes that the $n$-spike per burst solution destabilizes in a subcritical period-doubling bifurcation and the $(n+1)$-spike solution arises in a saddle-node bifurcation. It is clearly seen from Fig. 9 that the maximal number of coexisting states $N_{s}$ tends to grow with increasing spike number in the train. However, the fluctuation of $N_{s}$ is significant, and the whole plot looks quite random.

To understand how the number of synchronous regimes varies with $k_{S}$, let us consider the behavior of the effective coupling function as calculated for the seven-, eight-, and nine-spike trains (Fig. 10). We first note that the shape of the effective coupling function for $V$ coupling is much more complicated than for $S$ coupling. This is associated with the dynamics of the individual Sherman model where $V$ and $S$ are fast and slow variables, respectively. The spiking dynamics causes well-pronounced short-range oscillations of $\Gamma_{a}$ around zero. Another interesting observation is that a smooth 

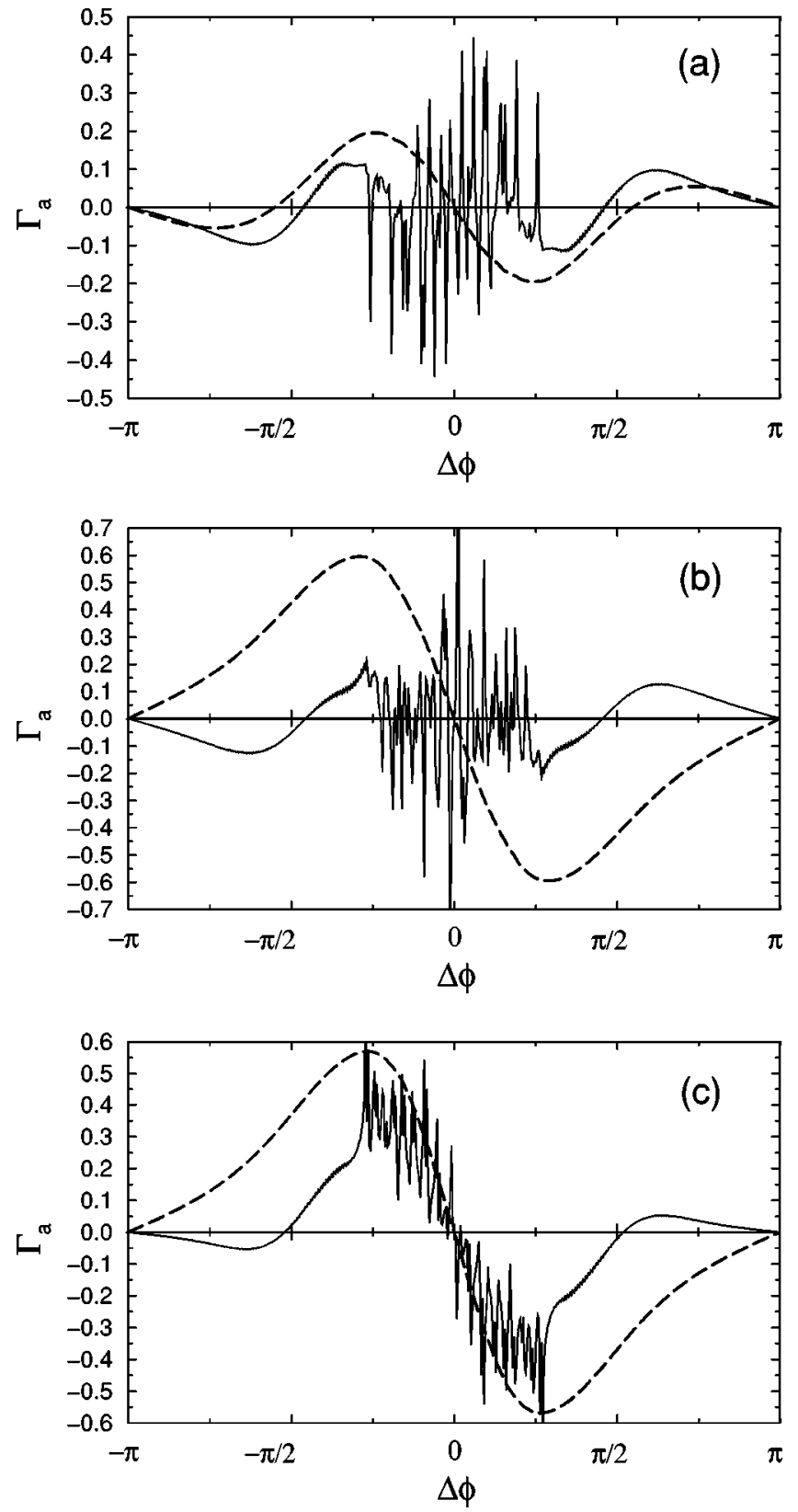

FIG. 10. Effective coupling function for the multispike bursting regimes. The solid line is for $V$ coupling while the dashed line is for $S$ coupling (a) seven spikes per train at $k_{S}=0.00011$; (b) eight spikes per train at $k_{S}=0.00009$; (c) nine spikes per train at $k_{S}$ $=0.00008$. Note, how the slow variation of $\Gamma_{a}$ in (c) causes the number of stable synchronization regimes to be quite small, even for $V$ coupling.

deformation of a long-range component of $\Gamma_{a}$ with varying $k_{S}$ (rather than changes in short-range oscillations of $\Gamma_{a}$ ) leads to changes of the number of intersections with zero. Inspection of Fig. 10(c) shows that the region of short-range oscillations of $\Gamma_{a}$ still exists but the long-range structure dominates. As a result, the number of stable synchronous states for the nine-spikes per train bursting dynamics is only four.

The behavior described here supports the hypothesis that the dephasing effect can play a significant role for the long-

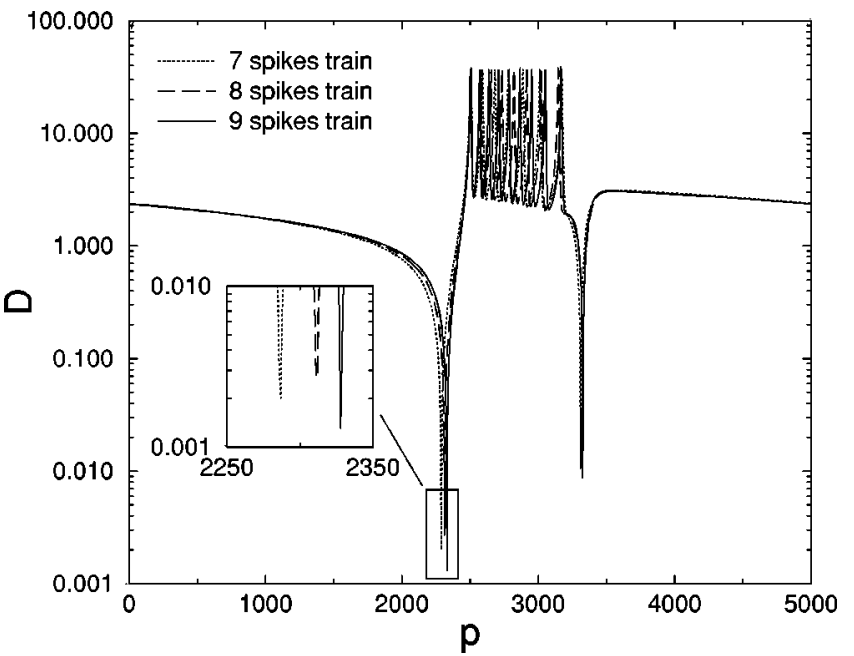

FIG. 11. Distance profile plotted against the position along the limit cycle for bursting oscillations with seven, eight, and nine spikes per train. Note that the minimal distances occur immediately before the bursting states, and that these distances vary nonmonotonically with the number of spikes per burst.

range variation of $\Gamma_{a}$ and, hence, cause the abrupt changes of the number of coexisting regimes. The strength of the dephasing effect can be indirectly measured by calculation of the minimal distance $D_{\min }$ between the limit cycle and the nearby equilibrium point. More detailed information can be achieved via a distance profile along the trajectory on a closed orbit (Fig. 11). To produce this figure, the orbit was divided into 5000 pieces of equal time interval $T / 5000$. The calculated distance $D$ was then plotted against the specified points on the orbit. Surprisingly, perhaps, the spiking region is not the closest to the equilibrium point. This means that adding spikes to a train with decreasing $k_{S}$ may not be so important as varying of the distance at the minimum point (see enlargement). Note that the distance at this point (being actually the minimal distance $D_{\min }$ ) changes nonmonotonically with decreasing $k_{S}$. Hence, dephasing can explain the irregular changes of the set of coexisting regimes. To find some correlation, we introduce the quality $N_{s} / N$ charactering how effectively the number of spikes in a train is transformed to the set of synchronous regimes. We compare the changes of this quality with the change of the minimal distance under variation of $k_{S}$. According to the simple quantitative analysis at the beginning of this section, one can expect that $N_{s} / N \approx 2.0-1 / N$ for the case of "perfect" bursting. In practice, the $N_{s} / N$ curve jumps within the range [0.666; 4.25]. Moreover, one can observe a certain correlation between curves for $N_{s} / N$ and for $D_{\min }$. This means that the distance variation between the limit cycle and equilibrium point rather than the number of spikes per train governs the phase multistability for the bursting regimes (Fig. 12).

\section{SUMMARY}

We analyzed a number of mechanisms that can cause or influence the occurence of phase multistability in two coupled bursters. Our main findings were the following.

To estimate the number of stable synchronous states for a 


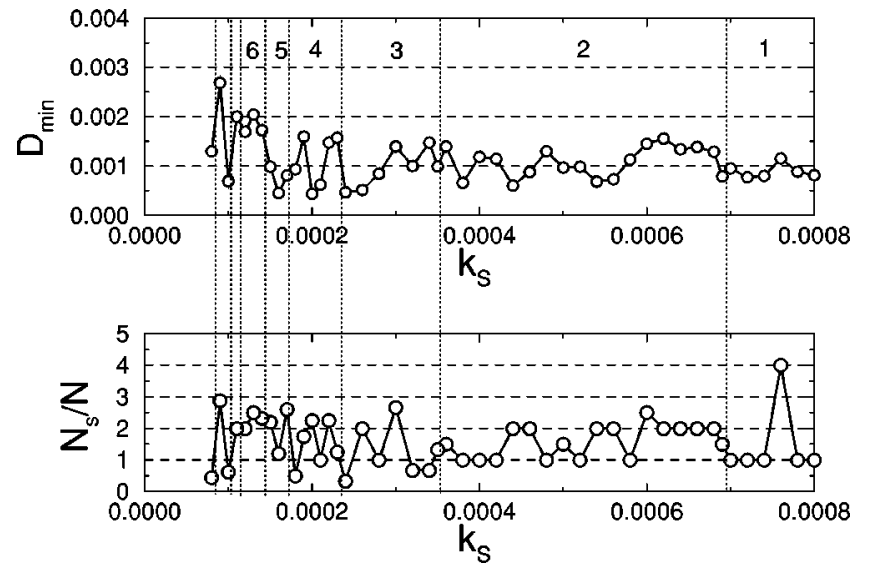

FIG. 12. There is a certain correlation between plots for the minimal distance from the equilibrium point to the limit cycle (upper panel) and for the number of coexisting stable regimes, normalized to the spike number per train (lower panel)

system of two weakly diffusively coupled bursting models it is not enough to know the wave forms of the oscillations in different regions of parameter space. Bursting oscillators often have specific phase portraits, involving regions of fast and slow motion, passing of trajectories close to singular points, etc. As a result, the dephasing effect can play an important role in the formation and evolution of coexisting regimes.

The minimal distance from a limit cycle to an equilibrium point was suggested as a diagnostic tool to estimate the strength of dephasing. We revealed an apparent correlation between the minimal distance and number of coexisting solutions.

A simple qualitative approach was introduced to count the expected number of synchronous regimes. According to this approach the number of such regimes should increase with increasing spikes per train. This approach works in the region of the period-doubling route to chaotic bursting. At a period-doubling bifurcation, a corresponding doubling of the stable synchronous regimes tends to take place, even when their original number was enchanced due to the dephasing effect.

Our analysis was based on the method of effective coupling which is valid for vanishingly weak diffusive coupling. However, we checked our results by direct numerical simulations for coupling strengths of order of $10^{-4}$. Thus, the effects we discussed are structurally stable.

Although the biophysical mechanisms underlying the bursting behavior may vary from cell type to cell type, we expect many of our findings to remain valid. However, the analysis leaves a number of open questions concerning the structure of the synchronization regions (Arnol'd tongues) for nonidentical cells as well as in the influence of a stronger coupling.

\section{ACKNOWLEDGMENTS}

This work was partly supported by INTAS (Grant No. 01-2061). O.S. acknowledges the Natural Science Foundation of Denmark, and S.M. asknowledges RFBR Grant No. 01-02-16709.
[1] X. -J. Wang and J. Rinzel, in The Handbook of Brain Theory and Neural Networks, edited by M. A. Arbib (MIT Press, Cambridge, 1995), pp. 686-691.

[2] H.A. Braun, H. Bade, and H. Hensel, Pfluegers Arch. 386, 1 (1980).

[3] H. A. Braun, H. Wissing, K. Schäfer, and M. C. Hirsch, Nature (London) 367, 270 (1994).

[4] R.E. Plant and M. Kim, Biophys. J. 16, 227 (1976).

[5] C. Morris and H. Lecar, Biophys. J. 35, 193 (1981).

[6] P.M. Dean and E.K. Matthews, J. Physiol. (London) 210, 255 (1970)

[7] S. Ozawa and O. Sand, Physiol. Rev. 66, 887 (1986).

[8] L.S. Satin and D.L. Cook, Pfluegers Arch. 414, 1 (1989).

[9] V.V. Astakhov, B.P. Bezruchko, E.N. Erastova, and E.P. Seleznev, Z. Tekh. Fiz. 60, 19 (1990) [Sov. Phys. Tech. Phys. 35, 1122 (1990)].

[10] T.E. Vadivasova, O.V. Sosnovtseva, A.G. Balanov, and V.V. Astakhov, Discrete Dyn. Nat. Soc. 4, 231 (2000).

[11] D.E. Postnov, T.E. Vadivasova, O.V. Sosnovtseva, A.G. Balanov, and E. Mosekilde, Chaos 9, 227 (1999).

[12] O.V. Sosnovtseva, D.E. Postnov, A.M. Nekrasov, E. Mosekilde, and N.-H. Holstein-Rathlou, Phys. Rev. E 66, 036224 (2002).

[13] R.H. Rand and P.J. Holmes, Int. J. Non-Linear Mech. 15, 387
(1980).

[14] D.G. Aronson, E.J. Doedel, and H.G. Othmer, Physica D 25, 20 (1987).

[15] S.K. Han, C. Kurrer, and Y. Kuramoto, Phys. Rev. Lett. 75, 3190 (1995).

[16] D.E. Postnov, S.K. Han, and H. Kook, Phys. Rev. E 60, 2799 (1999).

[17] G. de Vries, A. Sherman, and H.-R. Zhu, Bull. Math. Biol. 60, 1167 (1998).

[18] S.H. Park, S.K. Han, S. Kim, C.S. Kim, Sangwook Kim, and T.G. Kim, ETRI J. 18, 161 (1996).

[19] S.H. Park, S. Kim, H.-B. Pyo, and S. Lee, Phys. Rev. E 60, 2177 (1999).

[20] E. Mosekilde, B. Lading, S. Yanchuk, and Yu. Maistrenko, BioSystems 63, 3 (2001).

[21] Y. Kuramoto, Chemical Oscillations, Waves, and Turbulence (Springer-Verlag, New York, 1984).

[22] A. Sherman, J. Rinzel, and J. Keizer, Biophys. J. 54, 411 (1988).

[23] S. Yanchuk, Yu. Maistrenko, B. Landing, and E. Mosekilde, Int. J. Bifurcation Chaos Appl. Sci. Eng. 10, 2629 (2000).

[24] E. Mosekilde, Yu. Maistrenko, and D. Postnov, Chaotic Synchronization-Applications to Living Systems (World Scientific, Singapore, 2002). 